Document downloaded from:

http://hdl.handle.net/10251/139658

This paper must be cited as:

Araujo, A.; Rincon, J.; Carrascosa Casamayor, C.; Julian Inglada, VJ.; Novais, P. (03-2). Emotions detection on an ambient intelligent system using wearable devices. Future Generation Computer Systems. 92:479-489. https://doi.org/10.1016/j.future.2018.03.038

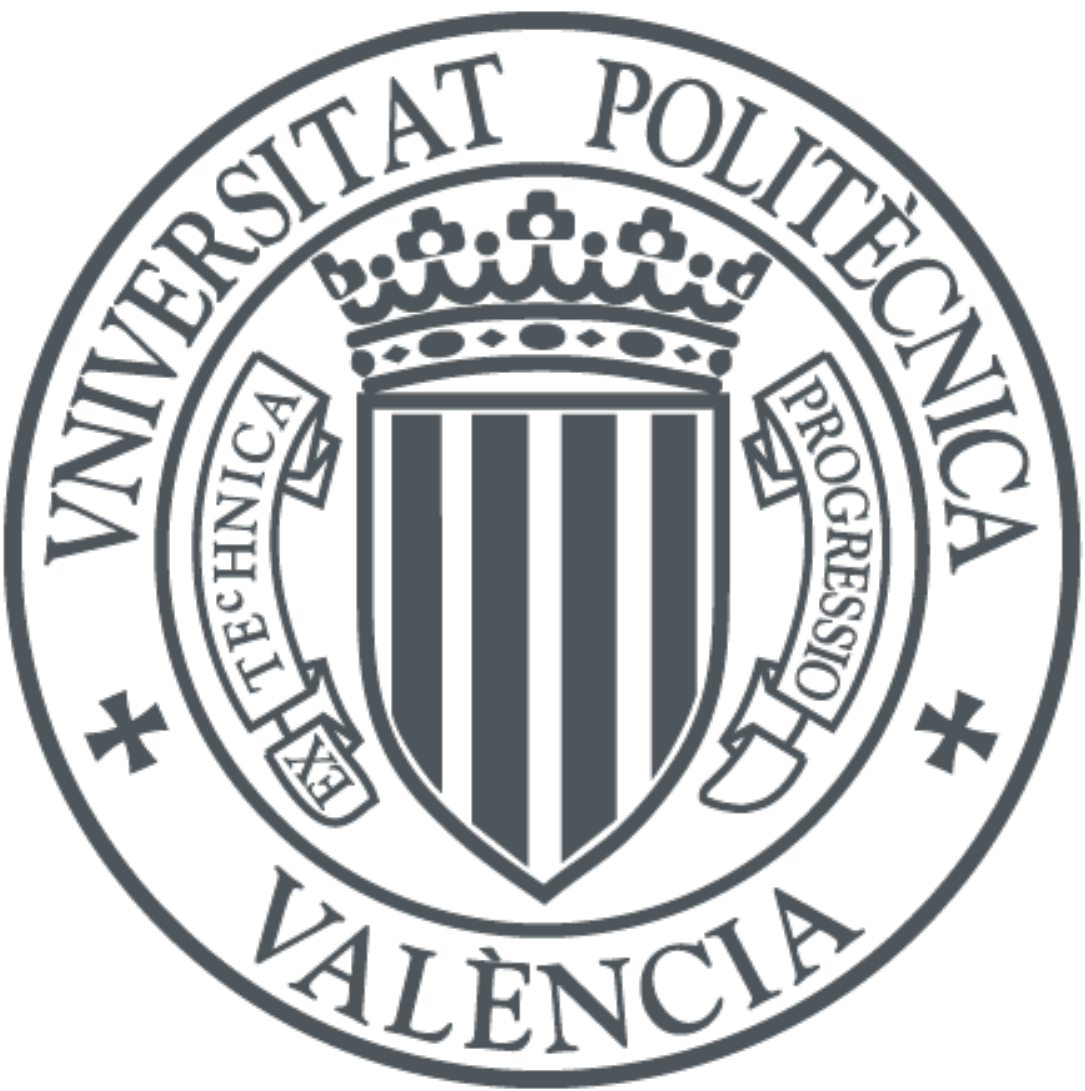

The final publication is available at

https://doi.org/10.1016/j.future.2018.03.038

Copyright Elsevier

Additional Information 


\title{
Emotions Detection on an Ambient Intelligent System Using Wearable Devices
}

\author{
Angelo Costa $^{\mathrm{a}, *}$, Jaime A. Rincon ${ }^{\mathrm{b}}$, Carlos Carrascosa ${ }^{\mathrm{b}}$, Vicente Julian ${ }^{\mathrm{b}}$, Paulo \\ Novais $^{\mathrm{a}}$ \\ ${ }^{a}$ Centro ALGORITMI, University of Minho, Braga (Portugal) \\ ${ }^{b}$ D. Sistemas Informáticos y Computación. Universitat Politècnica de València. Valencia \\ (Spain)
}

\begin{abstract}
This paper presents the Emotional Smart Wristband and its integration with the iGenda. The aim is to detect emotional states of a group of entities through the wristband and send the social emotion value to the iGenda so it may change the home environment and notify the caregivers. This project is advantageous to communities of elderly people, like retirement homes, where a harmonious environment is imperative and where the number of inhabitants keeps increasing. The iGenda provides the visual interface and the information center, receiving the information from the Emotional Smart Wristband and tries achieve a specific emotion (such as calm or excitement). Thus, the goal is to provide an affective system that directly interacts with humans by discreetly improving their lifestyle. In this paper, it is described the wristband in depth and the data models, and is provided an evaluation of them performed by real individuals and the validation of this evaluation.
\end{abstract}

Keywords: Multi-agent Systems; Emotional Agents; Internet of Things

* Corresponding author

Email addresses: acosta@di.uminho.pt (Angelo Costa), jrincon@dsic.upv.es (Jaime A. Rincon), carrasco@dsic.upv.es (Carlos Carrascosa), vinglada@dsic.upv.es (Vicente Julian), pjon@di.uminho.pt (Paulo Novais) 


\section{Introduction}

Ambient Assisted Living (AAL) is increasing rapidly and a large number of projects are being developed with the aim of providing assistance to elderly and disabled people $[1,2,3]$. What we are able to observe is that most of them have 5 as a goal to provide a simple interaction with the users with minimal effort. One common issue that most possess is that they rely on automatisms and static user profiles.

While they are effective in terms of simple tasks they do not encompass the ability of cater to changes that are part of the complex human states (like boredom), which changes the user profile [4].

The modulation of a user profile that emulates one's decision process must have the ability of representing emotional states $[4,5,6]$. The emotional states affect greatly the human decisions, where one decision to a specific situation may vary from one opposite to another if the current emotional state of a person 15 is happy or sad.

One way to address this issue is to ask the user every time for his or her consent to every action/decision, which defeats the purpose of being discrete and ubiquitous [6].

Another solution is using an adaptive system that is able to perceive these emotional changes. The information of these systems can be used to transparently support a decision according to one's emotional response. Moreover, emotional profiles may be created to provide common responses that were expected from the person that was emulated.

To capture the emotional status there are several ways that may be used, but one of the most discrete way is to monitor human physical changes. The way that humans perceive the world influences their emotional state and that has a repercussion on the physical level $[7,8]$.

There are signals that the human body inadvertently displays, like skin/muscle tensioning, pupil dilatation and micro-movements. Most of them have a correso sponding bio-electrical impulse, which in turn can be captured by sensors. 
In this paper we propose the usage of a wearable device in form of a wristband, named Emotional Smart Wristband (ESW) that sends information to the iGenda (an AAL platform), enabling it to schedule new tasks according the emotional status. Furthermore, the ESW plays an important part in Intelligent Virtual Environments, where the deployment environments can be simulated to test multiple outcomes of real interactions.

We also present in detail the composition of the ESW and the procedures and architecture of translating bio-signals into emotions using the PAD (Pleasure, Arousal, Dominance) concept and neural networks. We then establish the ground 40 work of the operational part of the system and how can the ESW contribute to other projects through giving real data or simulated scenarios in accordance to the collected data.

The objective is to create a complete AAL platform, using the ESW and the iGenda combined, that is able to cater to its users in a discrete way, and 45 gather emotional information that enriches the iGenda's knowledge about the user, such as likes and decisions. Thus, being able to forecast future decisions of these users, being able to implement them in virtual scenarios where they are tested and validated.

\section{1. $i G e n d a$}

iGenda is the base structure for communicating with the users. The aim of the iGenda is to manage the daily activities of the care-receivers and caregivers, using their agendas $[9,10]$. The intention is to streamline the interaction and to minimize the intrusion that forced interaction may require. iGenda uses a multiagent system that connects with the ESW and uses the care-receiver' emotional status to schedule/remove activities or events based on the feelings demonstrated, as well as send notifications to the caregivers about abrupt emotional changes. This way we are able to shape the users' environment as transparently as possible.

The use of the ESW has a motive: understanding how the user feels about the iGenda suggestions. iGenda contains several information about its users 60 and their responses but the issue is that these interactions may be biased. Our 
belief is that detecting the user's emotions we are able to obtain an unbiased response, thus being able to be more true to the users preferences and possibly adapt faster to them. Moreover, there are some extra features that the ESW has that can be used by the iGenda, like detecting heart problems or an heart attack and reporting the vital signs, which can be used to create health reports for the caregiver. iGenda has been extensively explained in previous articles $[11,9,10]$. Thus, in this paper our aim is to explain the ESW features and architecture, focusing on the interpretation of the emotions and how we translate electrical signals into emotions.

The paper is structured as follows: section 2 shows the related projects in the AAL and emotion detection areas; section 3 presents the ESW architecture, the emotional model and the virtual actor concept and the wristband components and operation; section 4 presents the evaluation of the proposed models and hardware with real subjects with the resulting data and its interpretation; finally, section 5 presents the conclusion and future developments.

\section{Related work}

The AAL area is currently very prolific, as it is object of various developments and novel projects. The AAL domain is very complex (providing healthcare services to elderly and mentally/physically challenged people) and presents an so important social strand that affects a numerous people, thus it is very relevant. AAL proposes systems and platforms that use technological helpers to perform daily tasks in a home environment. These helpers have the role of enhancing the task of the caregivers, providing extra help when the caregivers are not available and constantly monitoring the care-receivers for dangerous situations that they may encounter.

The Caregiver's Assistant project uses RFID and a database with activities and a fast inference mechanism that allows the identification actions being performed [12]. It uses RFID cards to track the users location and beacons that remotely read them, thus being able to locate accurately each user position. One 
issue with the hardware is that the beacons have a limited range, requiring that the users are in almost direct contact with them, thus they are very invasive and require effort to work and the users are aware of the procedures.

The CoME project [13] uses wearable sensors to monitor the platform users and smartphones to interact with them and to collect reports from them. Additionally, the platform has tutorials about how to perform certain activities that are showed to the caretakers to localize and access health reports from their assigned users. The platform aimed environments are elderly homes or similar places that care for a community of elderly people. The goal is to optimize the care provided by few caretakers to many care-receivers. Technologically, each deployed system is connected with the rest of the network and shares and receives information from the wearable sensors, adjusting constantly the services provided.

The ALADIN [14] allows the users to control the home environment (lights' brightness and colour) according to their emotional state. The users use a glove that reads biosignals (photoplethysmography), which transmits that information to the server, and according to their pulse/heart-rate the illumination is dimmed or colour changed. The goal of this project is to create comfort and peace by adjusting the environment to the user emotions. The glove limits the user to a confined space and it cannot get wet or be used to manage heavy objects. Thus it is very invasive, possibly biasing the results as the users are aware of apparatus and operation.

Pepper is a result of a private effort from the SoftBank company and is a servant humanoid robot [15]. Its aim is to interact with human beings, reading their emotions to create an empathic connection, and perform light tasks. It was designed to be a social companion, doing tasks like receiving people and provide useful information about a topic or task. The idea behind this robot was to create a companion for elderly people that was able to follow them around their home and proactively interact with them. The robot has a powerful processing unit and several sensors. It uses cameras and voice modulation to obtain the emotion status of the person that is interacting. Relying on imprecise sensors 
(cameras and microphones) means that the robot can only detect 4 emotions on exceptional environmental conditions (joy, sadness, anger and surprise). Those conditions are hard to achieve, thus, most of the times Pepper is unable to detect the emotions, being unreliable, and most of the time relying in artificial intelligence to show a probable emotion.

The works by $[16,17]$ introduce an architecture for emotion detection in smart environments (medical and residencies). The aim is to provide a system that is able to fuse multiple sources of information (physiological signals, facial expression and behaviour) to obtain the emotional state of the monitored users. With this information, the authors propose the regulation of sound (music), colour and light to achieve calm and happy emotions on the users. They have devised a wearable that is able to detect the electro-dermal activity and blood volume, which they measure the tensioning and relaxation of the body. Furthermore, with cameras they capture facial expressions and movement in the environment. With the facial expressions the application is able to capture directly the emotional state, and to compensate the natural unreliability of this process (that requires appropriate lightning, head position, distance, ...) a ceiling camera is available, to capture the movement of the users, detecting erratic movements and common patterns. The issue with these systems is the scalability problems and the fusion of distinct sensor systems. Albeit the authors use recent methodologies (like neural networks) to do the heavy lifting, correlating very distinct concepts (physical and psychological) may open the door to a high number of false positives due to the causation effects.

One common problem revealed of these projects is that they are interested in the implementation and the execution of their components but do not reveal any particular interest on the opinion of the users towards the devices that they are using.

Commonly, most of these projects overlook the opinion and emotions that the users have towards them. They may be liked by the users, and that does not pose a problem, but if they are disliked by the users it is very hard to know that information to improve them. Furthermore, in terms of personalization there is 
a reduced number of projects that tackle the perspective of using emotions to model services and visual interactions.

The recognition of emotions is a relatively easy task for a human: simply listening the nuance in the voice of a person or looking at her face. During the last few years, different methods for the automatic recognition of emotions have been studied. One of the best known techniques is based on the use of videos and images [18]. Moreover, the use of Electroencephalography (EEG) [19] approaches have provoked very significant improvements. This is because they allow direct measurement of the brain. However, we can find other studies the change in the diameter of the pupil, since this changes are related to human cognitive process. This techniques called Pupillometry [20] measure the pupil diameter when the people is exposed to a different stimuli. The pupillometry is used in different aspect as Language Processing, Memory and Decision Making, Perception, Cognitive Development and Emotion.

Nevertheless, these two approaches have drawbacks, video image requires a high processing load and the EEG is a very invasive method.

According to this last consideration, other human parameters have been considered lately, like body posture [21] or bio-signals from the body [22]. Regarding this last approach, bio-signals are a very interesting field of research to try to automatically recognize emotions in humans. This is due to the existence of a great amount of different signals that can be measured in a human body, e.g., Galvanic Skin Response (GSR), Electromyography (EMG), Electrocardiography (ECG), Electrodermal Activity (EDA), Blood Volume Pulse (BVP), Peripheral Temperature (SKT), and Respiration (RESP).

Due to the large number of existing bio-signals, one of the problems is the selection of the most appropriated bio-signals to be used as a method for the transformation of signals into emotions [23, 24]. Thus, for the measurement of bio-signals it is crucial to employ appropriate sensors and a great precision is needed to obtain good reliability.

Current approaches try to place different sensors on complex devices, and this way the device can measure from different sensor types and different sensors 
sources. Typically, these devices are called smart devices or smart sensors [25]. These devices also offer services to the rest of the system through some kind of connectivity functionality like bluetooth, wi-fi, ... In this sense, the devices are perceived by the rest of the entities of the system as a resource that offers services [26].

Next it is presented the ESW architecture, that tries to tackle the issues of the projects presented in this section.

\section{Emotional Smart Wristband}

This section describes the proposed platform which allows the schedule of new tasks according to the emotional status of the users. To do this, the platform is mainly formed by an Emotional Smart Wristband (ESW) and the iGenda system $[10,11,9]$. The ESW monitors the GSR and the Photoplethysmogram, and transforms it into an emotion classification.

With this information, the iGenda is able to use it to associate that emotion with the suggestions sent to the users. The goal is to synchronize the conscious decision with the subconscious one and obtain an unbiased classification based on the emotional response. The iGenda learning algorithms adjust the data to be in accordance to the user likes, improving the future suggestions, evolving along with the user. Moreover, iGenda is able to receive the information of multiple ESW and process them individually or in group. The reason behind the group emotion detection is that it is very useful to determine the global emotion status of an environment that is populated by multiple people, like a nursing home.

AAL promotes the individual as the most important element in the system, but that is applicable only when it is considered only one individual, which is not always the case. For instance, when we consider multiple individuals (like in nursing homes) we find that the best way to obtain a harmonious ambient is when most of the people tend to an overall emotion (considering varying degrees). The people in nursing homes behave socially, so there are natural 
forming groups with leaders and followers roles [27], thus the group is influenced by the leader decision even if the people individually do not like/agree with that decision. Tracking the groups emotional state is useful to understand the social bound between the users and to extract the real emotional response of each user even when influenced by the group. Thus, avoiding a very biased set of activities that the leader enjoys and the rest only tolerates. By using the ESW the users can respond unbiased of what their feelings are towards an activity. Tracking the community responses results in the overall likeness and in the social aspect identify if the leader is a good one or not.

\subsection{Architecture}

The ESW platform is mainly composed of a set of smart wristbands which monitor the emotional state of a group of people and transmit the information to the iGenda platform (see Figure 1). Moreover the ESW platform includes special agents that calculate the group emotion (or Social Emotion) of the participants, the Social Emotion Agent (SEtA), and resorting to machine learning techniques and makes it available for consumption [28, 29]. Moreover, the platform also includes an Intelligent Virtual Environment, where the deployment environments can be simulated through the employment of virtual actors.

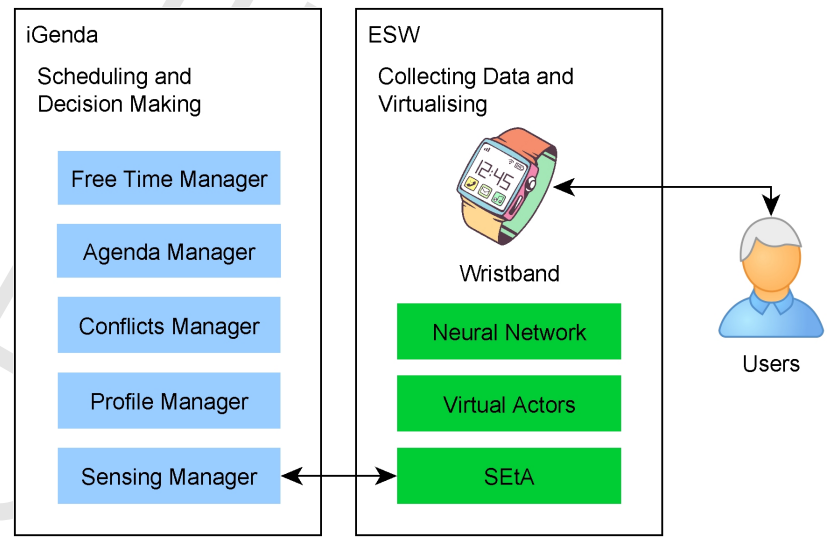

Figure 1: Integration between the ESW and the iGenda platforms. 
The voltage outputted from the sensors is captured locally (in the wristband) and pre-processed there, thus the information fed to the agents is in form of text or meta-data. Additionally, the multi-agent system has dedicated agents that grab that information and verify against the adopted models to extract the current emotion, joining it to the rest of the agents community that represent other users. The overarching result is the availability of the immediate emotion of each user and the global emotion of all users and the emotional trends and evolution. The trend of the group emotion is important as it is easier to find the culprits of the emotional changes.

For instance, consider a bar, it contains a group of friends and has music playing. Now consider that some of these friends have different musical tastes. If a music is playing that is enjoyable to $90 \%$ of the group we are able to notice an emotional progression towards "satisfied", while if it is the opposite (90\% dislikes) we can observe movement in the opposite direction. One of the possible aim of this analysis is obtaining a playlist that is enjoyable to most of the group with a specific alignment of songs that cater to each individual like. This specificity is used to avoid reaching a tension point where one or more persons have reached a saturation point and refuse any further change or abandon the environment.

The task of the iGenda is to consume this information and use its scheduling features to change the events of each participant, based on his/her profile, to achieve a specific emotion, thus guiding the group to a common emotional state. The emotional states are measured and placed in the PAD [30, 31] model, outputting a representation of the emotional state, easing the task of the iGenda on choosing new events. We have adopted the PAD model due to the simplicity and the datasets available. Furthermore, we are able to project the information captured into a $3 \mathrm{D}$ space, which facilitates the way the information is perceived.

\subsection{Background on physiology signals}

The signals exposed in this section are used by the wristband to detect human emotions, however we acquired an ECG signal to correlate the PPG signal. This correlation allows us to know if the peak of the ECG (complex QRS) was 
corresponding to that detected by the wristband (using PPG). However, another technique used is the Blood Volume Pulse (BVP). The BVP signals is derived of a PPG and is obtained using the PPG, the BVP measures the changes in blood volume in arteries and capillaries by shining an infrared light (a light-emitting diode) through the tissues. The infrared light reflected is captured by the PPG

Figure 2 shows three signals: (i) the blue signal corresponding to the GSR measure; (ii) the green signal corresponding to the ECG signal; and (iii) corresponding to the PPG signal. It is important to take into account that all the signal was acquired without any kind of filtering.

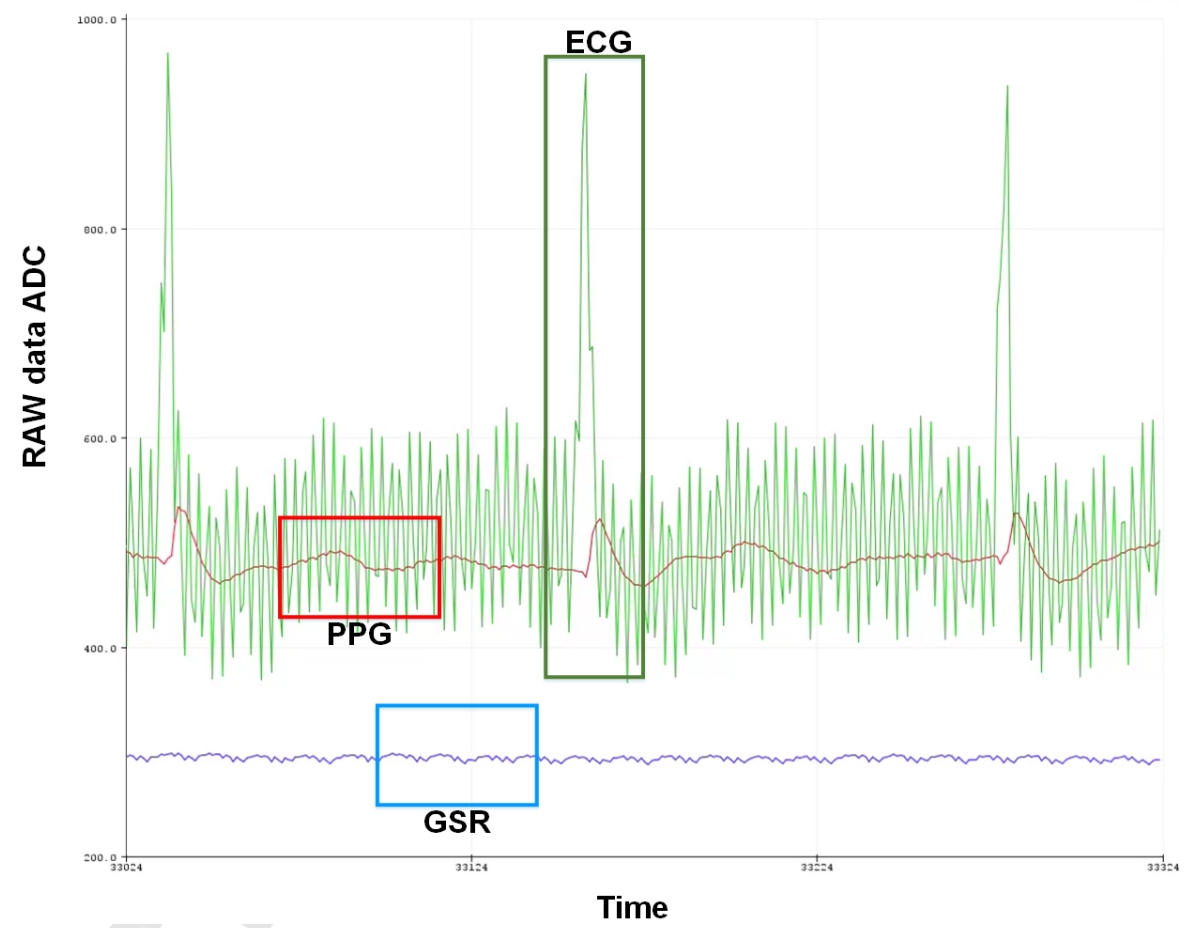

Figure 2: ECG, PPG, GSR bio-signals capture over time. 
The model seen in Figure 3 is a granular visual representation of the PAD model where the Valence replaces the Pleasure as in the psychological area is more representative and has a larger range of values [32]. The model has 12 sub-quadrants that are 30 degrees from each other. The emotion is represented by the vector $\vec{E}(A g)=[$ Arousal, Valence $]$ and the representation of emotions uses polar coordinates, constituted by the angle and the magnitude of the vector.

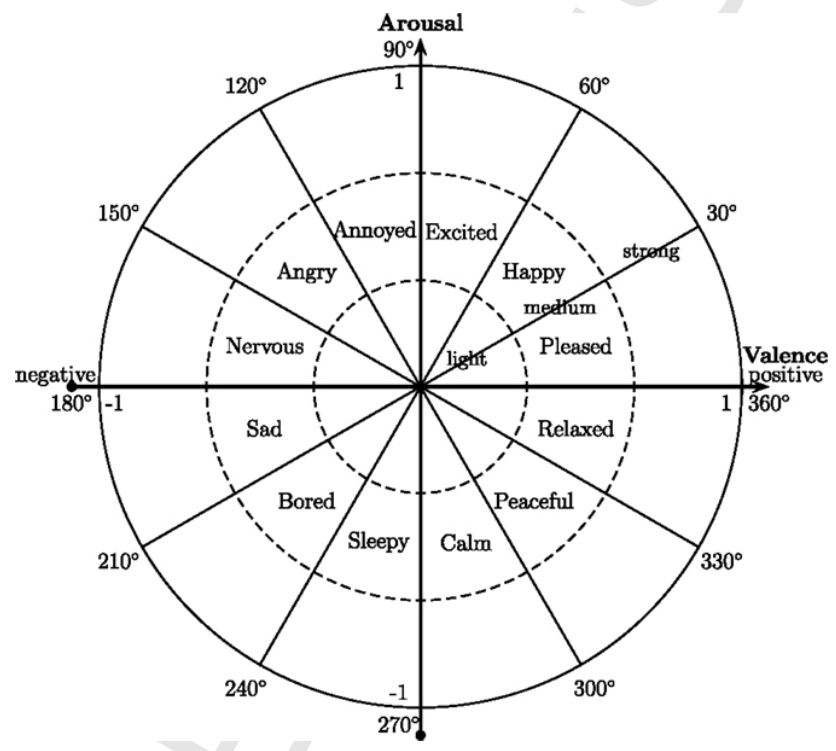

Figure 3: Emotions circle [33].

We use a fuzzy logic process that transforms qualitative values to quantitative values that are able to be placed in the model showed in Figure 3. Thereon, a neural network is trained using the DEAPdataset ${ }^{1}$ to achieve a real-world result from real humans. The correlation between the data captured and the dataset is established in the neural network, meaning that each processed electrical input can be translated into an emotion.

${ }^{1}$ http://www.eecs.qmul.ac.uk/mmv/datasets/deap/ 


\subsection{Virtual Actors}

A new development is the inclusion of virtual actors in the system that

\section{emulate the real participants. This feature aims to enhance the decision-making} process of the system, detecting in advance the possible emotional states and preparing changes to the participants surrounding environment to accommodate these changes and proactively shift them to other states if the expected state is not desirable [34, 35].

290 In this case specifically, when related to the elderly there is little margin for experimentation, as they have a large number of health issues (physical and/or cognitive disabilities) and are very fragile. The emotional profile helps to preview the possible reactions to changes on their daily routines and their daily emotion when performing specific activities. The group emotion is very useful in environments like nursing homes and residential elderly communities. In this type of environment is very common to perform group activities and to generate teams of elderly people. One common issue is the user verbal response and the real feeling, e.g., one "enjoyable" activity may be so due to peer pressure or to social engagement with the caregivers $[36,37]$. If the caregiver is able to receive unbiased information about how one feels about an activity it is easier to schedule similar or different activities or group that person with other people [38].

Another benefit from using virtual actors is the possibility of introducing Intelligent Virtual Environments (IVE). In this specific case, the IVE's would be used to project the environments where the users reside and to simulate all of the possible components, meaning that every sensor/actuator is mapped as an agent, thus it is able to directly interact with the human agents. This forwards our research by having safe environment enabled to test multiple outcomes of real interactions in fractions of second and project the optimal actions to achieve a certain outcome. 


\subsection{Wristband Description}

This section describes the main aspects of the proposed Emotional Smart Wristband. With the introduction of wearable devices in recent years in our society, a growing boom has begun in the design of smart bracelets. This is mainly due to the reduction of the size and manufacturing costs of the electronic components. Thanks to this, in recent years we have been able to observe intelligent bracelets that measure our oxygen saturation (SPO2), heart rate and monitor the way we sleep. However, it is possible to use these new technologies in order to detect our emotional states. This detection is performed using biosignals, such as those measured so far by commercial bracelets. However, it is necessary to add more signals, in order to obtain better detection and classification of emotions. Previous studies [39] demonstrated that variations in skin resistance could be associated with stress levels in humans. Other studies [5],[40] also shown that the heartbeat and body temperature could be associated with emotional changes. Based on these studies, we have designed a bracelet able to capture and process biosignals, with the aim of detecting emotional states and thus creating applications in IoT and AAL areas. The proposed bracelet incorporates four sensors, with which four basic emotions are detected in the circumplex model [41] (Happy, Angry, Sad and Relaxed). To detect emotional states present in the circumplex model, the designed wristband incorporates the following sensors:

- EDA (see Figure 4a) - for measuring the changes in the electrical conductance of the skin. This includes GSR, which results from sympathetic neuronal activity. EDA is a sensitive psychophysiological index of changes in the autonomic sympathetic excitation which is used for the detection of the emotional and cognitive states [42].

- Bluno Ble V1.0² (see Figure 4b) - the microcontroller. It has low consumption bluetooth communication (BLE). It has an 8-bit Atmega328 CPU

\footnotetext{
${ }^{2}$ https://www.dfrobot.com/
} 
and is compatible with Android 4.3+ devices with BLE4.0 and iOS 7.0+ devices.

- Temperature sensor (see Figure 4c) - for measuring the irradiated temperature as infrared light that our body emits, according to our moods.

- Photoplethysmography sensor (see Figure 4d) - for measuring the variations in blood flow. Using these variations it is possible to obtain the heart rate, which have been used in the detection of stress levels [43].

- Inertial Measurement Unit (IMU) (see Figure 4e) - a combination of accelerometer and a gyroscope. The accelerometer measures the acceleration of the wrist in the three axes $[\mathrm{X}, \mathrm{Y}, \mathrm{Z}]$. The gyroscope allows us to measure the angles of rotation of the wrist and the angular speed.

In order to integrate these sensors into a wristband, several designs were made to improve the position of the sensors and minimize the size. The final ESW prototype can be seen in Figure 5. The IMU and the microcontroller are above another board containing the rest of the sensors.

From controlled experiences (explained in detail below), we have deduced that these sensors allows us to determine the emotional state of an individual. However, not all humans respond in the same way to external stimuli. For this reason, it is necessary to customize the bracelet. This personalization will help to reliably detect the emotional state of each person who uses it. To perform this customization an experiment has been designed in which the subject will be exposed to a series of visual stimuli (images). In each of the sessions the subjects will wear the bracelet that continuously captures the physiological variations. Also, the subjects will be monitored through a camera, which performs a facial analysis of their facial response to the stimuli. Through this facial response, we get additional information about the emotion expressed by the subjects. The usage of cameras will only be used on this personalization procedure.

The images used to stimulate emotional changes have been obtained from the 


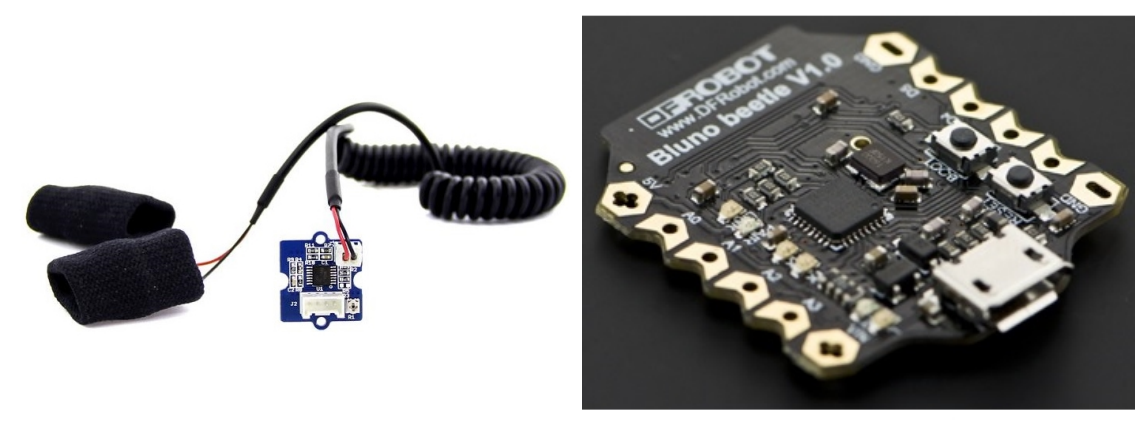

(a) EDA

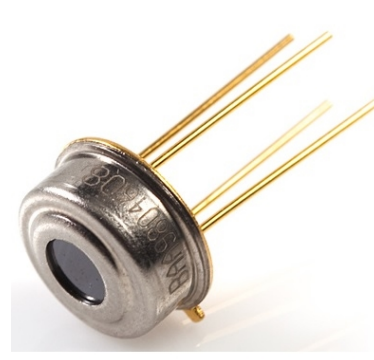

(c) Infrared Temperature

Sensor

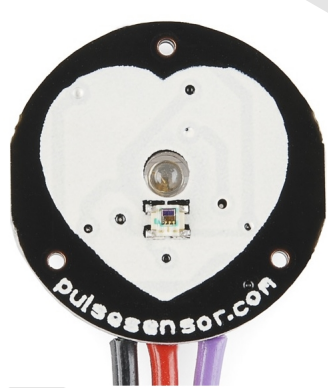

(d) Photoplethysmography sensor (b) Bluno

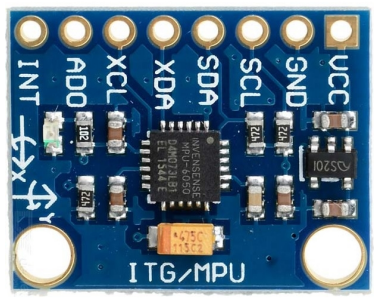

(e) IMU

Figure 4: Sensors used to acquire the different biosignals.

Center for the Study of Emotion and Attentions of the University of Florida ${ }^{3}$ [44].

This center has created a database formed by a series of images, sounds and texts, previously catalogued. In our experiments, we used the International Affective Picture System (IAPS). This system has been developed to provide a set of normative emotional stimuli for experimental research on emotion and attention. In this dataset there is a large set of standardized, emotionally evocative and internationally accessible color photographs, including contents across a wide

\footnotetext{
${ }^{3}$ http://csea.phhp.ufl.edu/index.html
} 


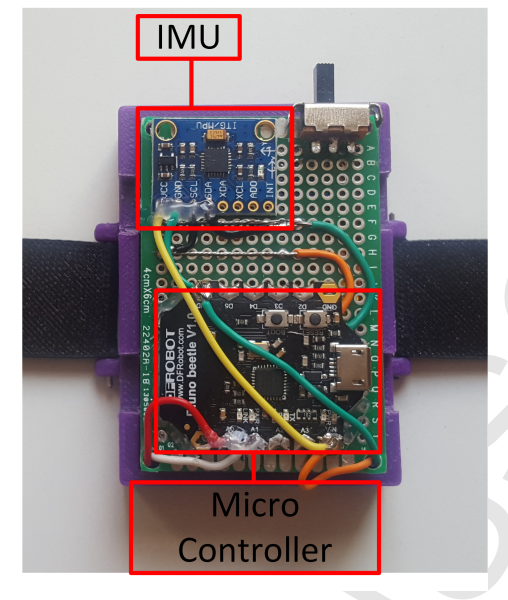

Figure 5: ESW final prototype.

range of semantic categories. In the process of personalizing the bracelet, the user is only exposed to these visual stimuli (images). The information obtained as a result of the exposure of these stimuli is stored in a database. This database is structured as follows: [ID_image, Emotion_Detected, Bio_Signals, SAM].

The first field is the identifier of the image of the IAPS database, this will allow us to know the emotion that represents that image or sound. The second field is the emotion detected through the camera, this detection is done through the Microsoft ${ }^{4}$ API. This API classifies human emotions in eight classes: Anger, Contempt, Disgust, Fear, Happiness, Neutral, Sadness and Surprise. The third field is an array of ten dimensions, six of which are of the IMU and the remaining three correspond to the biosignals acquired by the wristband. These biosignals will be captured for 20 seconds, during this time the user look at the pictures. In our tests we use 644 images to create the training dataset and 538 images to do the test. Once has been finished the time, the user will perform a SelfAssessment Manikin (SAM) [45] test (see Figure 6), which corresponds to the last field in our database. SAM is a non-verbal pictographic evaluation technique

\footnotetext{
${ }^{4}$ https://www.microsoft.com/cognitive-services/en-us/emotion-api
} 

that directly measures the pleasure, arousal and dominance associated with a person's affective reaction to a wide variety of stimuli. It is important to take into account that the images don't represent an emotion and the value of PAD is extracted of SAM test. The emotion detected using the Microsoft API is used to check a correlation of the emotion detected by a visual system with the
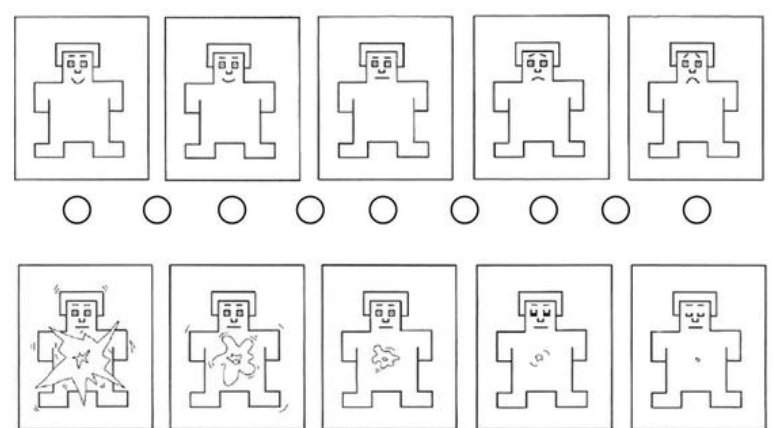

$\bigcirc \bigcirc$
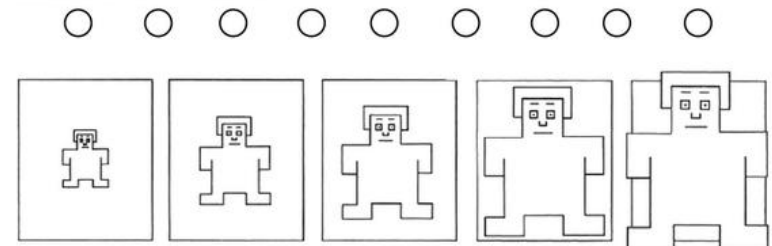

$\bigcirc \bigcirc \bigcirc$ ○

Figure 6: Self-Assessment Manikin (SAM) test.

In summary, for each obtained signal, a vector which includes the values of the previously presented equations is formed. Once the data has been obtained for each of the users, a customized emotion classification model is trained. To perform this training, the techniques of descriptive statistics have been adopted, as suggested by Picard et al [46] to perform the extraction of characteristic vectors. For our experiments we have extracted the six following variables:

1. The means of the raw signals

2. The standard deviations of the raw signals

3. The means of the absolute values of the first differences of the raw signals 
4. The means of the absolute values of the first differences of the normalized signals

5. The means of the absolute values of the second differences of the raw signals

6. The means of the absolute values of the second differences of the normalized signals

Each feature vector has 18 elements because six features are extracted for each signal. This input vector is used by a neural network in order to classify the different emotional states of each test subject. There are different methods to perform this classification, but the most used is neural networks.

In our experiments, we have used Backpropagation. Our neuronal network consists of 18 input neurons, 100 neurons in the middle layer and 7 neurons in the output layer (one for each emotion: Angry, Disgusted, Fearful, Happy, Sad, Surprised, and Neutral) (see Figure 7). In order to obtain the best results (low value of final recognition error), different experiments were performed leading to improve the number of neurons in the middle layer. After several iterations, it was concluded that with 100 neurons in the middle layer were enough. These 100 neurons allowed us to obtain the best results when classifying the emotions.

\section{Evaluation}

Experiments were performed to 20 test subjects, using a database with 1182 images. This database was divided into two sets: the training set of 900 images, and the test set of 282 images.

The tests were done using the following protocol (see Figure 8):

1. The set of training images (50) was observed by the test subjects for 10 seconds. During these 10 seconds the signals of GSR, PPG, temperature and heart rate were recorded and stored.

2. At the same time, the subject was recorded, which was used to detect the emotion expressed by the subject. This detection was performed 


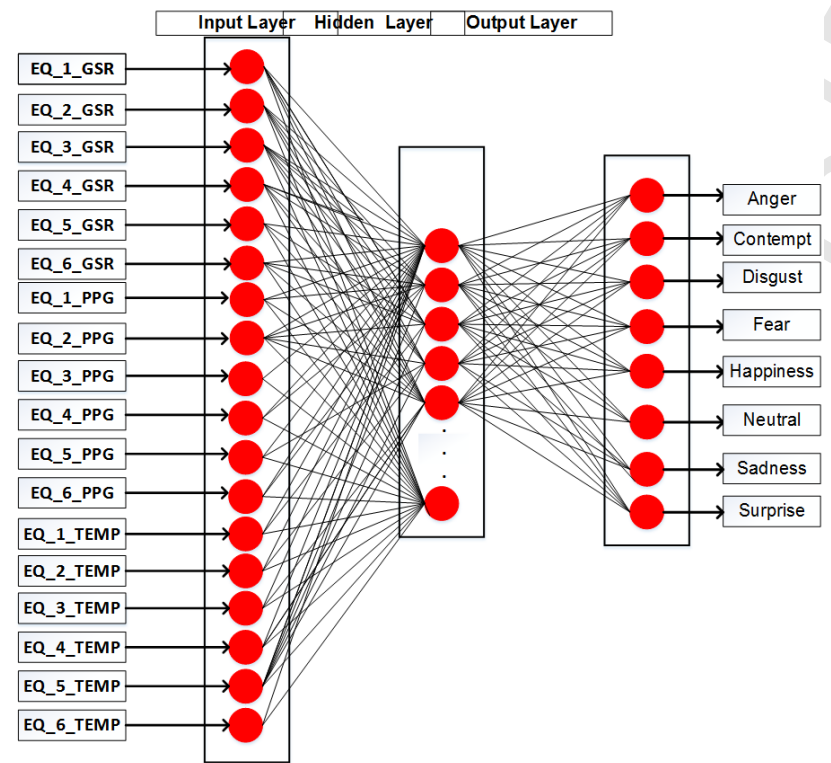

Figure 7: Neural network architecture.

using the Microsoft Detect Emotions Service, which detects the following emotions: Anger, Contempt, Disgust, Fear, Happiness, Neutral, Sadness and Surprise.

3. After 10 seconds of observation of the stimulus, the subject has 10 seconds more to respond to the SAM test.

Figure 9 shows the signals captured by the wristband, these strips are excerpts of signals recorded in the experiment when the user is exposed to the stimulus. It is important to note that the units of the signals are the same: $\mathrm{mV} / \mathrm{s}$. In the case of the temperature sensor, a temperature-to-voltage conversion was performed to have the three signals with the same unit. This conversion was performed using Equation 1, which is given by the manufacturer of the sensor. In the equation, $V_{i r}$ is the raw data of the IR channels, To is the temperature of the object, $T A$ is the ambient temperature and $A$ is the general sensitivity.

$$
V_{i r}=A\left(T o^{4}-T a^{4}\right)
$$




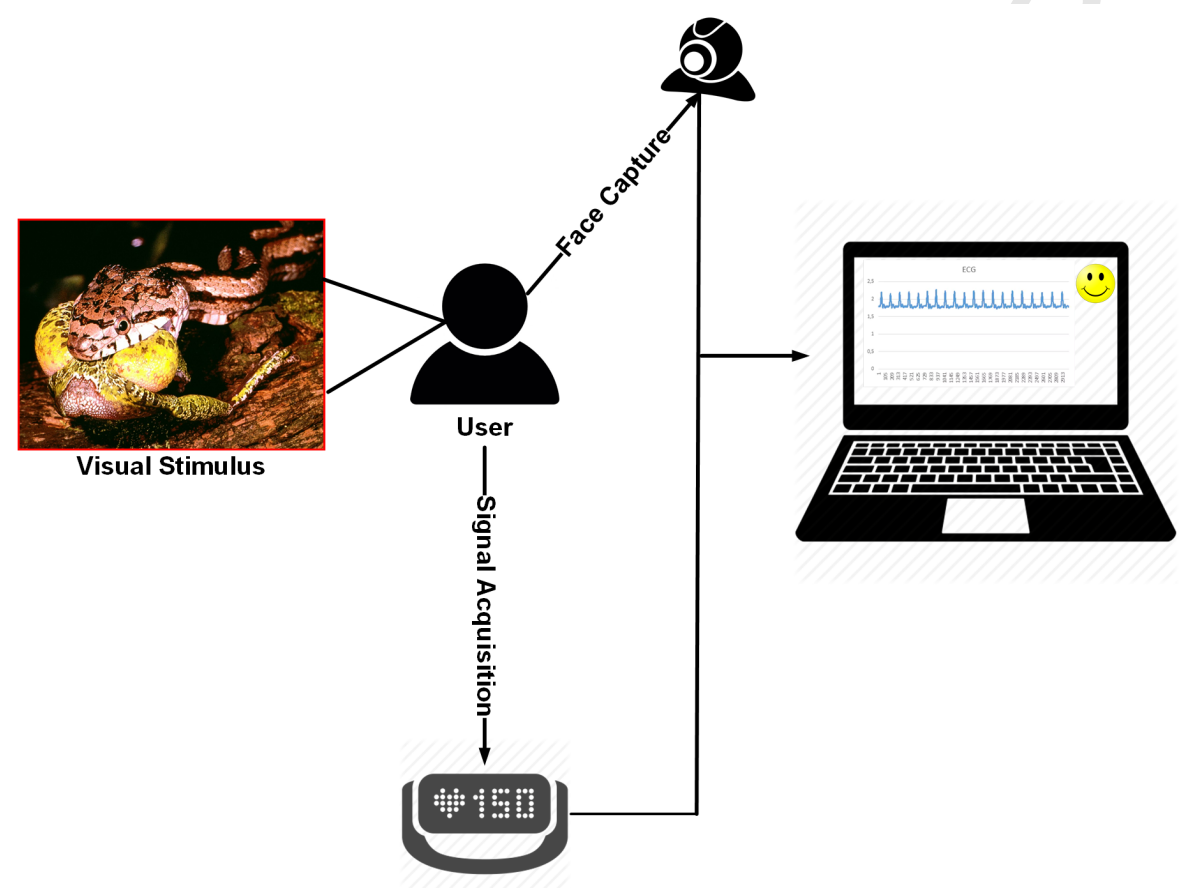

Figure 8: Description of the data capture process.

This neural network was trained using 18 features vector. However, our dataset has two outputs the first one corresponding to the emotion detected for the Microsoft API and the second one is the emotion obtained using the SAM test. The output using to supervise the neural network training is the result obtained through the Microsoft API, the SAM test give us a qualitative description emotion associated to the image.

In order to determine the best CNN architecture, the group of 20 subjects was divided into 4 groups of 5 subjects. Each network (corresponding to a group) was modified as the activation function of the middle layer and the output layer. In the same way, the momentum and learning rate was modified. Table 1 describes the groups and their parameters.

Mean square errors obtained in the experiments (see Figures 10a, 10b, 10c, 10d), allowed us to determine what could be the best neural network architecture 


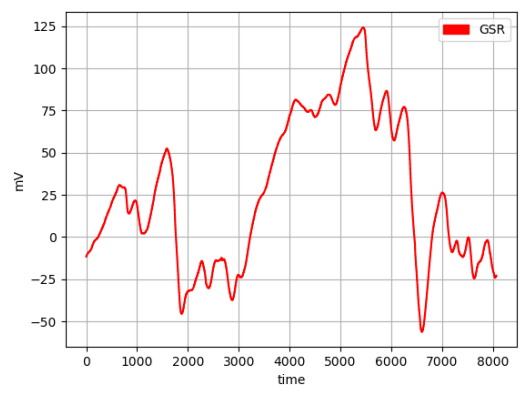

(a) GSR Signal.

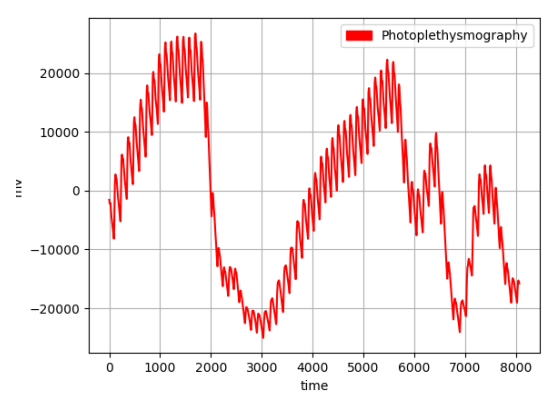

(b) Photoplethysmography Signal.

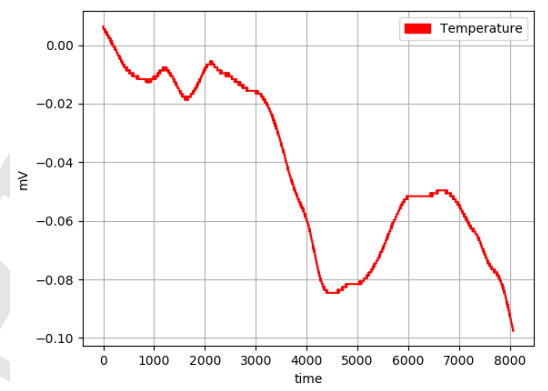

(c) Temperature Signal

Figure 9: Signals captured by the wristband.

to use. It is necessary to emphasize that it is possible to have many combinations

of architectures and factors that affect the learning of the neural network. Based on the graphs and classification positions obtained in each experiment, it was 
Table 1: Testing different configuration parameter for the neural network.

\begin{tabular}{|l|c|c|c|c|}
\hline & Medium layer activation function & Output layer activation function & Momentum & Learning Rate \\
\hline Test Subject 1-5 & TanhLayer & SoftmaxLayer & 0.1 & 0.1 \\
\hline Test Subject 6-10 & SoftmaxLayer & TanhLayer & 0.01 & 0.01 \\
\hline Test Subject 11-15 & LSTMLayer & SoftmaxLayer & 0.1 & 0.0001 \\
\hline Test Subject 16-20 & LSTMLayer & LinearLayer & 0.00001 & 0.1 \\
\hline
\end{tabular}

determined that the best architectures were those of subjects Subject 6-10 and Subject 11-15. However, it is important to clarify that there may be many combinations in this type of experiments. It is for this reason that, in future works, the test subjects will be given a personality test to group the subjects by personality similarity, thus being able to assign a neural network architecture according to their personalities.

After the evaluation process, we have obtained a CNN that allows us to estimate the emotional state of the wristband's users with a very low error. This error is related directly with the emotion prediction using the wristband and in our experiments we have achieved $20 \%$ of misclassification cases. The integration of this ESW response in the proposed platform will allow to manage the community emotion of a set of users (for instance, people in retirement homes) and to send this information to the care-receivers or caregivers through the iGenda system.

\section{Conclusions}

This paper has presented a new Emotional Smart Wristband (ESW) platform that allows capturing and communicate time-stamped bio-signals from a user to be translated to the current emotion he/she feels.

The goal of this platform is twofold: first, it has been designed to be integrated in the iGenda platform, to be used to adjust iGenda platform decisions to changes in the emotions not only of one agent, but of a group (as the platform allows to work with the concept of Social Emotion). The other goal is to record the emotions of a user and have the adjusted response to different stimuli, thus having a proper ESW response. This allows us to have a virtual representation 


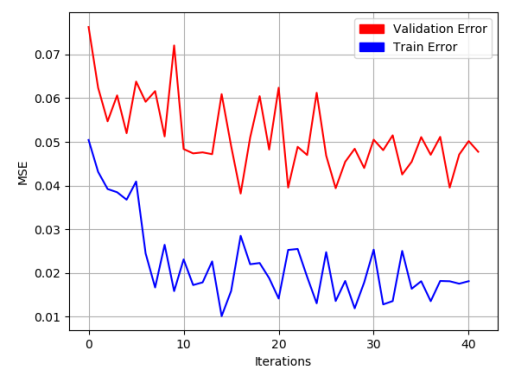

(a) MSE Test Subject 1-5.

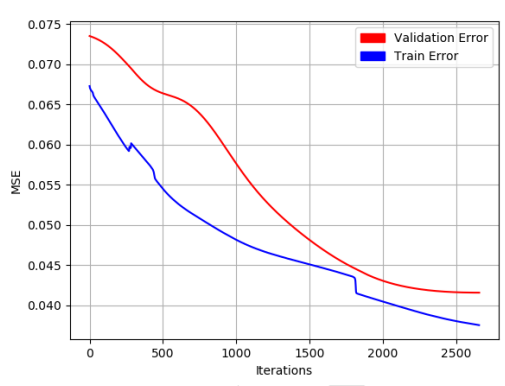

(c) MSE Test Subject 11-15

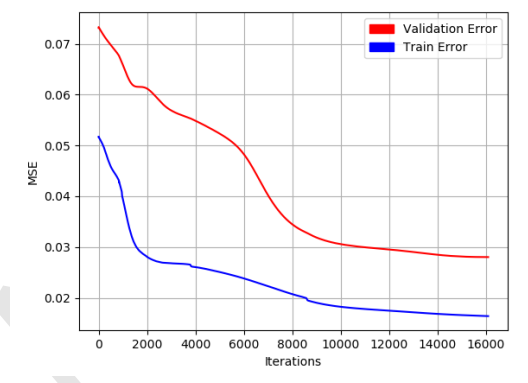

(b) MSE Test Subject 6-10.

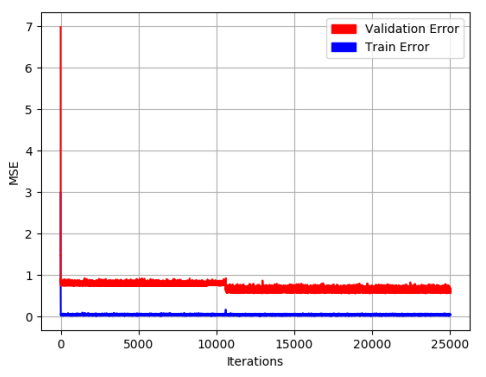

(d) MSE Test Subject 16-20

Figure 10: MSE representation of the test subjects. 
of the user that can be used in an intelligent virtual environment simulation.

This will allow preparing the settings of tasks and environmental conditions to be used in the iGenda and test them with the subjects without bothering them with changes or avoiding changes that would lead to distasteful experiences (typically a happy social emotion).

Only the settings carrying the simulation to the goal emotion would be carried out in the iGenda system interacting with the real world.

One of the strengths of this platform is the usage of a recommender process (through the iGenda) that helps an often forgotten population. Most of the projects are not directly aimed at the general population. Furthermore, the platform has discreet sensor systems, as well as more invasive systems for increased accuracy, although with a properly trained model we are able to use just the wristband, being very unobtrusive.

The modularity that this platform has is also an interesting strength, being possible several applications to the ESW ecosystem apart from the healthcare area, like: crowd control, visual interfaces management, stress and fatigue monitoring, virtual environments societies, among others.

\section{Acknowledgements}

This work is supported by COMPETE: POCI-01-0145-FEDER-007043 and FCT - Fundação para a Ciência e Tecnología within the projects UID/CEC/00319/2013 and Post-Doc scholarship SFRH/BPD/102696/2014 (Angelo Costa) This work is partially supported by the MINECO/FEDER TIN2015-65515-C4-1-R and the FPI grant AP2013-01276 awarded to Jaime-Andres Rincon.

\section{References}

[1] J. Bravo, D. Cook, G. Riva, Ambient intelligence for health environments, Journal of Biomedical Informatics 64 (2016) 207-210. doi:10.1016/j.jbi . 2016.10.009. 
[2] C. Ramos, Ambient Intelligence - A State of the Art from Artificial Intelligence Perspective, in: J. Neves, M. F. Santos, J. M. Machado (Eds.), Progress in Artificial Intelligence, Vol. 4874 of Lecture Notes in Computer Science, Springer Berlin Heidelberg, Berlin, Heidelberg, 2007, pp. 285-295. doi:10.1007/978-3-540-77002-2\{_\}24.

[3] D. J. Cook, J. C. Augusto, V. R. Jakkula, Ambient intelligence: Technologies, applications, and opportunities, Pervasive and Mobile Computing 5 (4) (2009) 277-298. doi:10.1016/j ·pmcj . 2009.04.001.

[4] J. Gratch, S. Marsella, Tears and fears, in: Proceedings of the fifth international conference on Autonomous agents - AGENTS'01, Association for Computing Machinery (ACM), 2001. doi:10.1145/375735.376309.

[5] C. Maaoui, a. Pruski, Emotion recognition through physiological signals for human-machine communication, Cutting Edge Robotics (2010) 317333doi: 10.5772/10312.

[6] J. van Hoof, H. Kort, P. Rutten, M. Duijnstee, Ageing-in-place with the use of ambient intelligence technology: Perspectives of older users, International Journal of Medical Informatics 80 (5) (2011) 310-331. doi:10.1016/j. ijmedinf .2011.02.010.

[7] S. Jerritta, M. Murugappan, R. Nagarajan, K. Wan, Physiological signals based human emotion recognition: a review, in: 2011 IEEE 7th International Colloquium on Signal Processing and its Applications, IEEE, 2011. doi:10.1109/cspa.2011.5759912.

[8] J. Wagner, J. Kim, E. Andre, From physiological signals to emotions: Implementing and comparing selected methods for feature extraction and classification, in: 2005 IEEE International Conference on Multimedia and Expo, IEEE, 2005. doi:10.1109/icme.2005.1521579.

[9] A. Costa, V. Julián, P. Novais, Advances and trends for the development 
of ambient-assisted living platforms, Expert Systems (2016) n/a-n/aEXSYMar-15-075.R1. doi:10.1111/exsy.12163.

[10] J. A. Rincon, A. Costa, P. Novais, V. Julián, C. Carrascosa, A dynamic emotional model for agent societies, in: Advances in Practical Applications of Scalable Multi-agent Systems. The PAAMS Collection: 14th International Conference, PAAMS 2016, Sevilla, Spain, June 1-3, 2016, Proceedings, Vol. 9662, Springer International Publishing, 2016, pp. 169-182. doi:10.1007/978-3-319-39324-7_15.

[11] A. Costa, P. Novais, R. Simoes, A caregiver support platform within the scope of an ambient assisted living ecosystem., Sensors (Basel, Switzerland) 14 (3) (2014) 5654-5676. doi:10.3390/s140305654.

[12] K. P. Fishkin, B. Jiang, M. Philipose, S. Roy, I sense a disturbance in the force: Unobtrusive detection of interactions with RFID-tagged objects, in: UbiComp 2004: Ubiquitous Computing, Springer Berlin Heidelberg, 2004, pp. 268-282. doi:10.1007/978-3-540-30119-6_16.

[13] Come, accessed on 2017-04-07 (2017).

URL htp://come-aal.eu

[14] E. Maier, G. Kempter, ALADIN - a magic lamp for the elderly?, in: Handbook of Ambient Intelligence and Smart Environments, Springer Berlin Heidelberg, 2010, pp. 1201-1227. doi:10.1007/978-0-387-93808-0_44.

[15] Pepper - softbank (2017).

URL https://www.ald.softbankrobotics.com/en/robots/pepper

[16] A. Fernández-Caballero, A. Martínez-Rodrigo, J. M. Pastor, J. C. Castillo, E. Lozano-Monasor, M. T. López, R. Zangróniz, J. M. Latorre, A. Fernández-Sotos, Smart environment architecture for emotion detection and regulation, Journal of Biomedical Informatics 64 (Supplement C) (2016) $55-73$. 
[17] J. C. Castillo, Á. Castro-González, A. Fernández-Caballero, J. M. Latorre, J. M. Pastor, A. Fernández-Sotos, M. A. Salichs, Software architecture for smart emotion recognition and regulation of the ageing adult, Cognitive Computation 8 (2) (2016) 357-367. doi :10.1007/s12559-016-9383-y. URL https://doi.org/10.1007/s12559-016-9383-y

[18] Y. Sun, N. Sebe, M. S. Lew, T. Gevers, Authentic emotion detection in realtime video, in: Computer Vision in Human-Computer Interaction, Springer Berlin Heidelberg, 2004, pp. 94-104. doi : 10.1007/978-3-540-24837-8_ 10.

[19] Y. Liu, O. Sourina, M. K. Nguyen, Real-time EEG-based Emotion Recognition and its Applications, in: Transactions on Computational Science XII, Vol. 6670, Springer, 2011, pp. 256-277. doi:10.1007/978-3-642-22336-5.

[20] S. Sirois, J. Brisson, Pupillometry, Wiley Interdisciplinary Reviews.

[21] M. Coulson, Attributing emotion to static body postures: Recognition accuracy, confusions, and viewpoint dependence, Journal of nonverbal behavior 28 (2) (2004) 117-139.

[22] F. Canento, A. Fred, H. Silva, H. Gamboa, A. Lourenço, Multimodal biosignal sensor data handling for emotion recognition, in: Sensors, 2011 IEEE, IEEE, 2011, pp. 647-650.

[23] J. Kim, E. André, Fusion of multichannel biosignals towards automatic emotion recognition, in: Multisensor Fusion and Integration for Intelligent Systems, Springer, 2009, pp. 55-68.

[24] A. Haag, S. Goronzy, P. Schaich, J. Williams, Emotion recognition using bio-sensors: First steps towards an automatic system, in: ADS, Springer, 2004, pp. 36-48.

[25] G. C. M. Meijer, C. M. Meijer, C. M. Meijer, Smart sensor systems, Wiley Online Library, 2008. 
[26] E. Munera, J.-L. Poza-Lujan, J.-L. Posadas-Yagüe, J.-E. Simó-Ten, J. F. B. Noguera, Dynamic Reconfiguration of a RGBD Sensor Based on QoS and QoC Requirements in Distributed Systems, Sensors 15 (8) (2015) 1808018101.

[27] P. A. Estabrooks, K. J. Munroe, E. H. Fox, N. C. Gyurcsik, J. L. Hill, R. Lyon, S. Rosenkranz, V. R. Shannon, Leadership in physical activity groups for older adults: A qualitative analysis, Journal of Aging and Physical Activity 12 (3) (2004) 232-245. doi:10.1123/japa.12.3.232.

[28] J. A. Rincon, V. Julián, C. Carrascosa, Representing social emotions in MAS, in: Y. Demazeau, K. S. Decker, J. B. Pérez, F. de la Prieta (Eds.), Advances in Practical Applications of Agents, Multi-Agent Systems, and Sustainability: The PAAMS Collection - 13th International Conference, PAAMS 2015, Salamanca, Spain, June 3-4, 2015, Proceedings, Vol. 9086 of Lecture Notes in Computer Science, Springer, 2015, pp. 308-311. doi : 10.1007/978-3-319-18944-4_34.

[29] J. A. Rincon, V. Julián, C. Carrascosa, An emotional-based hybrid application for human-agent societies, in: Á. Herrero, J. Sedano, B. Baruque, H. Quintián, E. Corchado (Eds.), 10th International Conference on Soft Computing Models in Industrial and Environmental Applications, SOCO 2015, Burgos, Spain, June 2015, Vol. 368 of Advances in Intelligent Systems and Computing, Springer, 2015, pp. 203-213. doi:10.1007/ 978-3-319-19719-7_18.

[30] A. Mehrabian, Analysis of affiliation-related traits in terms of the PAD temperament model, The Journal of Psychology 131 (1) (1997) 101-117. doi: $10.1080 / 00223989709603508$.

[31] A. Nanty, R. Gelin, Fuzzy controlled PAD emotional state of a NAO robot, in: 2013 Conference on Technologies and Applications of Artificial Intelligence, Institute of Electrical and Electronics Engineers (IEEE), 2013. doi:10.1109/taai.2013.30. 
[32] R. Gupta, Y.-J. Hur, N. Lavie, Distracted by pleasure: Effects of positive versus negative valence on emotional capture under load., Emotion 16 (3) (2016) 328-337. doi:10.1037/emo0000112.

[33] R. E. Thayer, The biopsychology of mood and arousal, Oxford University Press, 1989.

[34] H. S. Minhat, R. Mohd Amin, Sociodemographic Determinants of Leisure Participation Among Elderly in Malaysia, Journal of Community Health 37 (4) (2011) 840-847.

[35] J. M. Hartgerink, J. M. Cramm, T. Bakker, A. M. van Eijsden, J. P. Mackenbach, A. P. Nieboer, The importance of multidisciplinary teamwork and team climate for relational coordination among teams delivering care to older patients, Journal of Advanced Nursing 70 (4) (2014) 791-799.

[36] G. Fernández-Mayoralas, F. Rojo-Pérez, P. Martínez-Martín, M.-E. PrietoFlores, C. Rodríguez-Blázquez, S. Martín-García, J.-M. Rojo-Abuín, M.-J. Forjaz, Active ageing and quality of life: factors associated with participation in leisure activities among institutionalized older adults, with and without dementia, Aging \& Mental Health 19 (11) (2015) 1031-1041.

[37] D. Muñoz, F. Gutierrez, S. F. Ochoa, N. Baloian, Enhancing social interaction between older adults and their families, in: International Workshop on Ambient Assisted Living, Springer, 2013, pp. 47-54.

[38] P.-J. Chang, L. Wray, Y. Lin, Social relationships, leisure activity, and health in older adults, Health Psychology 33 (6) (2014) 516-523. doi: $10.1037 /$ hea0000051.

[39] M. V. Villarejo, B. G. Zapirain, A. M. Zorrilla, A stress sensor based on galvanic skin response (GSR) controlled by ZigBee, Sensors (Switzerland) 12 (5) (2012) 6075-6101. doi:10.3390/s120506075.

[40] F. Nasoz, K. Alvarez, C. L. Lisetti, N. Finkelstein, Emotion recognition 650 from physiological signals using wireless sensors for presence technolo- 
gies, Cognition, Technology \& Work 6 (1) (2004) 4-14. doi:10.1007/ s10111-003-0143-x.

[41] J. A. Russell, A circumplex model of affect, Journal of Personality and Social Psychology 39 (1980) 1161-1178.

[42] H. D. Critchley, Review: Electrodermal responses: What happens in the brain, The Neuroscientist 8 (2) (2002) 132-142, pMID: 11954558. doi: $10.1177 / 107385840200800209$.

[43] Firstbeat Technologies Ltd., Stress and Recovery Analysis Method Based on 24-hour Heart Rate Variability (2014) 1-13.

${ }_{660}^{6}$ [44] P. J. Lang, M. M. Bradley, B. N. Cuthbert, International affective picture system (IAPS): Affective ratings of pictures and instruction manual, Tech. Rep. A-8, The Center for Research in Psychophysiology, University of Florida, Gainesville, FL (2008).

[45] M. Bradley, P. J. Lang, Measuring Emotion: The Self-Assessment Semantic Differential Manikin and the, Journal of Behavior Therapy and Experimental Psychiatry 25 (I) (1994) 49-59. arXiv:0005-7916(93)E0016-Z, doi: 10.1016/0005-7916(94)90063-9.

[46] R. Picard, E. Vyzas, J. Healey, Toward machine emotional intelligence: analysis of affective $\backslash$ nphysiological state, IEEE Transactions on Pattern Analysis and Machine Intelligence 23 (10) (2001) 1175-1191. doi:10.1109/ 34.954607. 
Jaime Andre Rincon was born in Buga (Valle), Colombia, in 1978. He received the B.E. degree in biomedical engineering from the University Manuela Beltran, Colombia, in 2008, and the MS degree in Artificial Intelligence from Universitat Politècnica de València. He received his PhD on Computer Sciences at the Universitat Politècnica de València in 2018. As a researcher his interest is in multi-agent systems, robotics and emotional agents.

Angelo Costa has received his European Doctorate title at the University of Minho (Portugal) in 2013. He has also received his Master's title in 2009 by the University of Minho. Previously he worked as an Invited Assistant Professor at the Polytechnic of Porto, more specifically at the School of Management and Technology. As a researcher his interest is in multi-agent systems, ambient intelligence, mobile computing, machine learning, virtual organizations and privacy and data protection. He has participated in different research projects focused on Ambient Intelligence and Information Analysis both at a European and national level such as AAL4ALL, TIARAC. Additionally, he has been member of the organizing and scientific committee of several international symposiums such as PAAMS, ISAmI, PRO-VE, IDC, etc.

Vicente Julian holds a position of Full Professor of Computer Science at the Universitat Politècnica de València (UPV) where he has taught since 1996. Vicente Julian is member of the GTI-IA research group, and Deputy Director of the Computer Science Department at the UPV. Four international projects, two international excellence networks, twenty-one Spanish projects and four technology transfer projects have covered the research on Artificial Intelligence. He has more than 60 works published in journals with outstanding positions in the list of the Journal Citation Reports, or published in conference proceedings that have a system of external peer review and dissemination of knowledge comparable to journals indexed in relevant positions. Moreover, he has more than 130 contributions and a h-index of 27 . Vicente Julian has supervised 9 PhD Thesis.

Carlos Carrascosa was born in Valencia (Spain) and received the M.S. degree in Computer Science from the Universidad Politécnica de Valencia (UPV) in 1995. He obtained his Ph.D. in the Departamento de Sistemas Informáticos y Computación at UPV and is currently a lecturer involved in teaching several Al-related subjects at the UPV. His research interests include MAS, social emotions, consensus in MAS, Intelligent Virtual Environments, learning, serious games, information retrieval, and real-time systems. 
Angelo Costa

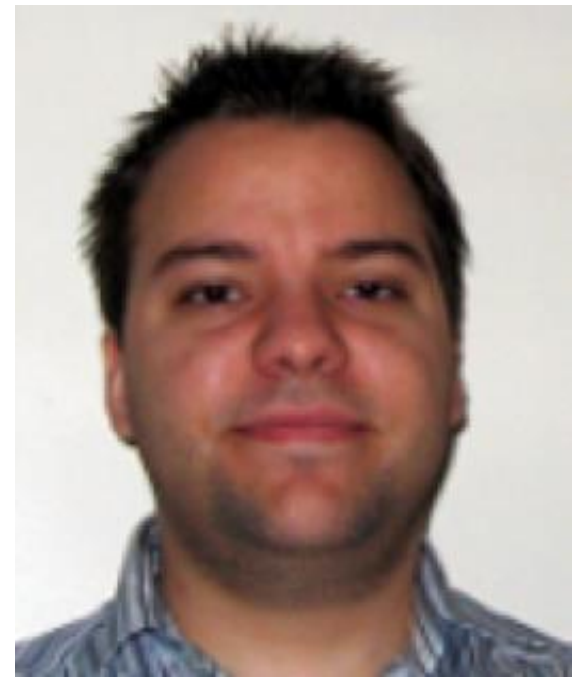

Jaime Andre Rincon

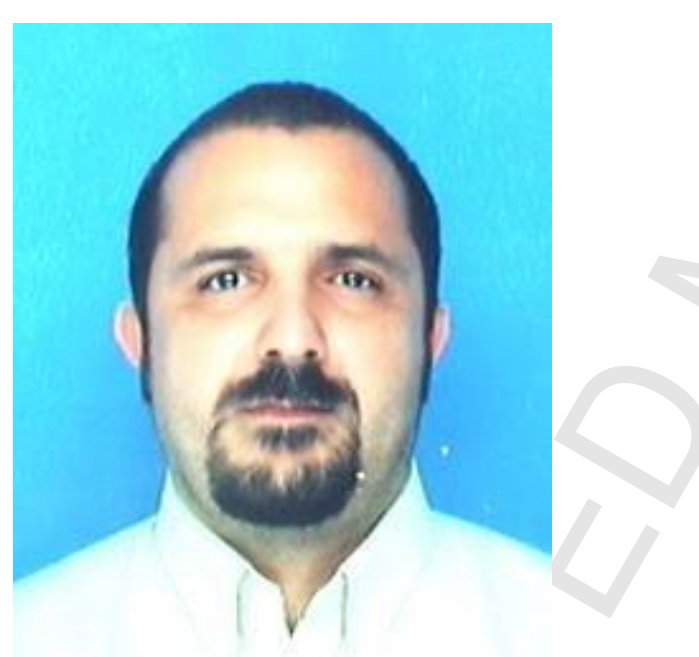


Carlos Carrascosa

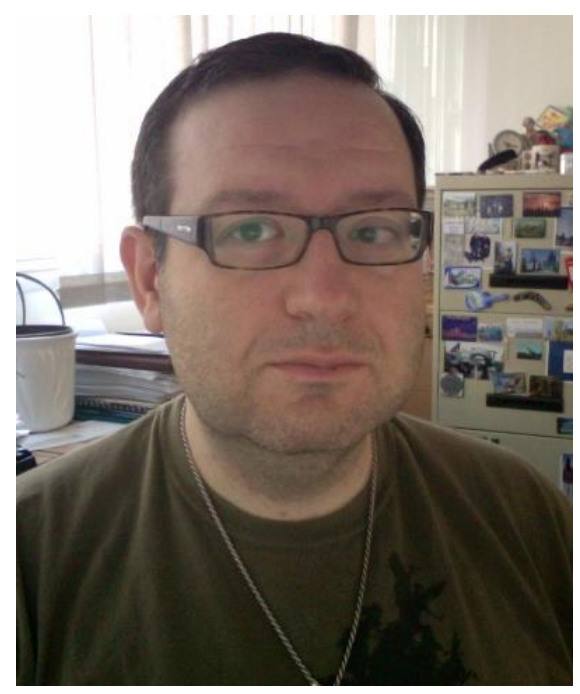

Vicente Julian

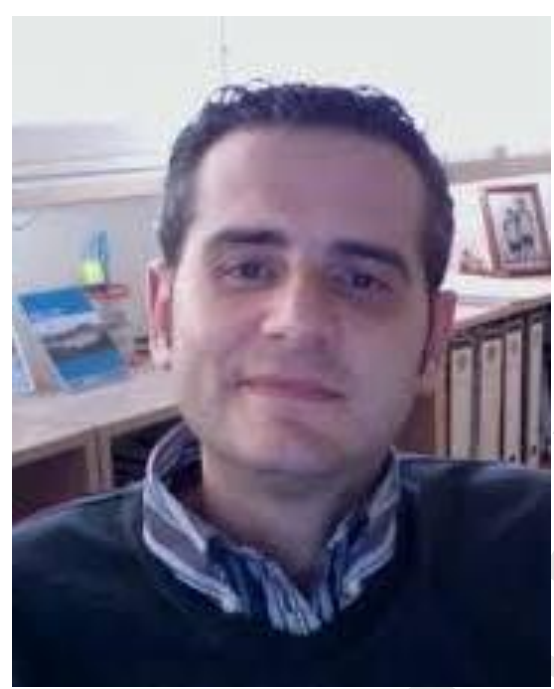

